\title{
Fat and Fat Free Mass Index Reference Percentiles of Healthy Turkish Children and Adolescent in Turkey
}

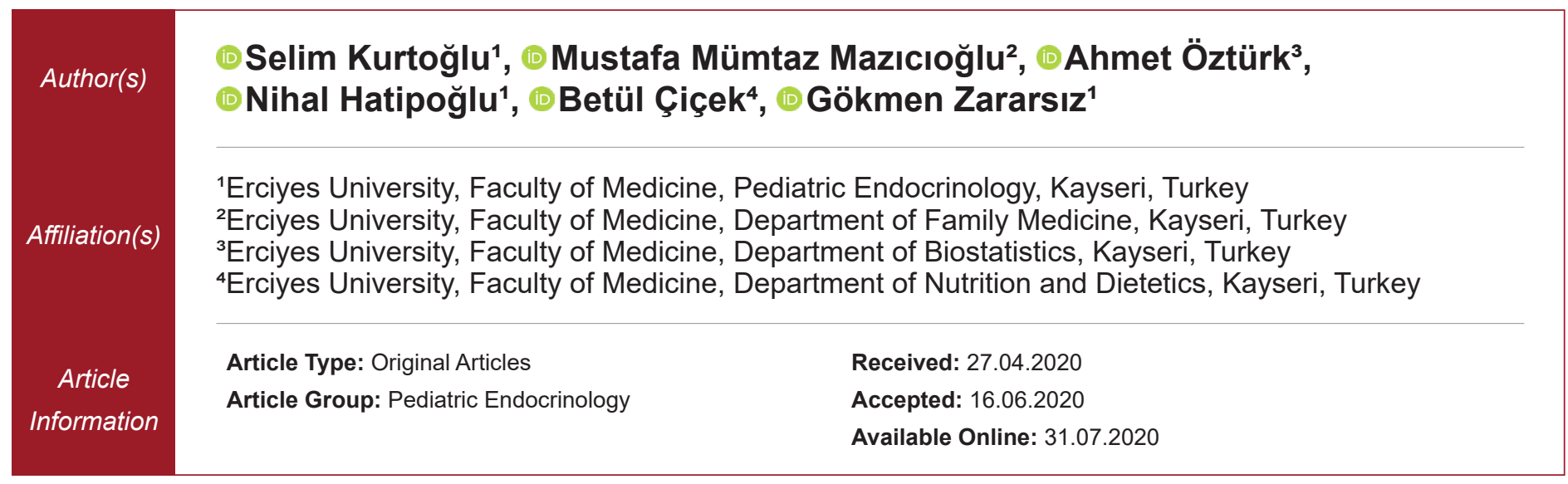

Cite this article as: Kurtoğlu S, Mazıcıoğlu MM, Öztürk A, Hatipoğlu N, Çiçek B, Zararsız G. Fat and Fat Free Mass Index Reference Percentiles of Healthy Turkish Children and Adolescent in Turkey. J Pediatr Acad 2020; 1(1): 8-15.

\section{Abstract}

In addition to body mass index (BMI), fat mass (FM), fat-free mass (FFM), and their index may be used to predict metabolic health risks. The aim of this study is to define age- and genderspecific FM, fat mass index (FMI), FFM and fat-free mass index (FFMI) percentiles for heatly Turkish children and adolescents. A total of 4028 (2252 girls, 1776 boys) participant aged 6-17 years were recruited. The body composition was evaluated by bioelectrical impedans. FM, FMI, FFM and FFMI percentiles were produced. FM, FFM, FMI and FFMI percentiles were calculated. FMI and FM were female predominance through 6 to 17 years. The differences in $3^{\text {rd }}-97^{\text {th }}$ percentiles of FFMI were $4.06-7.20 \mathrm{~kg} / \mathrm{m}^{2}$ respectively for males, where this difference was $4.06-6.95 \mathrm{~kg} / \mathrm{m}^{2}$ for females. We checked the age-specific contribution of FMI, FFMI to $\mathrm{BMI}$ and fat\% with Hattori chart and found that children with similar BMI may lie in different fat\%. Since FM and FFM are important for the evaluation of body composition, in addition to $\mathrm{BMI}$ and body fat\%, FM and FFM percentiles are required as local reference. Therefore, this study provides normative data for body FM, FMI, FFM and FFI percentiles.

Keywords: Fat mass, fat mass index, fat-free mass, fat-free mass index, children, adolescents

Correspondence: Nihal Hatipoglu, Erciyes University, Faculty of Medicine, Pediatric Endocrinology, Kayseri, Turkey

E-mail: nihalhatipoglu@yahoo.com 


\section{Introduction}

In addition to secular change in growth, change in body composition should also be considered as an indicator of endocrine and cardiometabolic parameter which needs serious concern. ${ }^{1}$ Since the onset of obesity has shifted to an early age, the complication of overweight and obesity in adults can also be observed in childhood. ${ }^{1-5}$

Early onset overweight and obesity in childhood is not only an endocrine and cardiometabolic disorder which are precursors of cardiovascular disease, hypertension, insulin resistance, diabetes mellitus, dyslipidemia, polycystic ovary syndrome, hyperandrogenism, neoplasm but also a serious cause of adult overweight and obesity burden. Other than endocrine and cardiometabolic problems social and emotional impairment extending to adulthood may even be a cause of non physical disorders. ${ }^{6-8}$

The primary assesment tools for nutritional status can be listed as height, weight, mid uppper arm circumference, and skinfold thickness. ${ }^{9}$ To diagnose the obesity-and overweight-related problems, body mass index (BMI) is still the fundamental method but not the competent measure, since BMI does not solely is a measure of body composition which discriminates fat and fat-free mass content. ${ }^{10}$ Then, we need non-complicated and useful measures to detect the main risk factors for endocrine and cardiometabolic risk such as fat mass or fat mass distribution. Since both fat mass (FM) and fatfree mass (FFM) constitutes BMI the interchanging ratio of fat mass and fat-free mass as an indicator of health risk that $\mathrm{BMI}$ does not discriminate. ${ }^{11,12}$ Failure of discriminating fat and lean mass for BMI lead to research of other methods like bioelectrical Impedance Analysis (BIA) and Dual-energy X-ray absorptiometry (DEXA). ${ }^{13}$

DEXA provides a precise assessment of FM (fat mass), FFM (fat free mass) and bone mineral density which are the three main body components. However, DEXA uses an X-ray source, which is not preferable for children and it is expensive and required specialized equipment. Thus it is not feasible for routine clinical practice (14). On the other hand, BIA method can also give valuable information about body composition. ${ }^{15}$

Although relatively small differences may exist between BIA and DEXA, these differences may be neglected since BIA is a practical, non-invasive and cheap method versus DEXA. ${ }^{9,16}$

The primary aim of this study is to produce the FM and FFM percentiles and fat mass and fat- free mass indexes (FMI, FFMI) in a quite competent sample in
Turkish children and adolescents for the first time. This would provide the opportunity of both to detect fat and fat- free mass and also its variation for height. Calculated percentiles then may be used in evaluation of body composition for thinness, overweight and obesity resulted by body fat or muscle mass content and distribution.

\section{Materials and Methods}

The Ethical Committee of Erciyes University, Faculty of Medicine, approved this study (Approval date: 03.04.2019/304, number: 04-01/168).

\section{ighlight}

-Although BMI is the most frequently used parameter in diagnosis, in certain circumstances BMI may not The use of BIA may help in fat mass and lean body mass that puberty precious, delayed puberty, overweight and obesity.
This is the most recent (2007-2008) and the comprehensive crosssectional study which uses the data of Determination of Anthropometric Measures of Turkish Children and Adolescents (DAMTCA II). ${ }^{17}$ The study sample consists of 1776 male and 2252 female $6-17$ years old children and adolescents.

In 6-17 years old children and adolescents age and gender specific FM, FMI, FFM and FFMI percentiles are produced by LMSP method (17). The device Tanita BC418MA (Tanita Corporation, Tokyo, Japan) was used to calculate fat mass (FM), fat-free mass (FFM) fat. The mass index (FMI; FM/height ${ }^{2}$ ), fat-free mass index (FFMI: FFM/ Height $^{2}$ ) were then calculated. ${ }^{16}$

Age-related FFM, FFMI, FM and FMI z-score plots were examined and the discontinuities were checked. Further, liberal cut-off values, where the z-scores of data values outside ${ }^{6,11}$, were used to detect outliers. ${ }^{18}$ After outlier detection, remaining 4.028 observations (1.776 boys, 2.252 girls) were randomly split into training (70\%) and validation $(30 \%)$ sets. Training set was used for model building and validation set was used for model validation and model selection. GAMLSS models were used to fit the models. ${ }^{19}$ For each anthropometric measure and each gender, three methods including LMS, LMST and LMSP methods were applied to data. Box-Cox normal (BCN), Box-Coxt (BCT) and Box-Cox power exponential (BCPE) distributions were used in GAMLSS models, respectively. Maximum penalized likelihood method was used, RS algorithm and Fisher scoring procedure was applied to estimate distribution parameters. As smoothing functions, cubic splines were used. Each gender was modeled separately. Analyses were conducted using GAMLSS package (version 4.3-1) of $R 3.1 .1$ software (www.r-project.org).

\section{Parameter Optimization}

In order to apply LMSP method, we followed the three step optimization procedure of Rigby and Stasinopoulos ${ }^{20}$ and generalized Akaike's information criteria with parameter \#3 for model selection. Firstly, identity link functions were defined for $\mu$ and $v$, log-link functions were defined for $\sigma$ and $\mathrm{T}$. An initial age transformation was optimized 
as $x=$ age $\lambda$ after a grid search of $\lambda$ between -2 to 2 in steps of 0.25 . Next, initial degrees of freedom of all four parameters was set to 1 and $\operatorname{df}(\mu), \lambda$ and $\operatorname{df}(\sigma)$ values were optimized respectively. For $\mathrm{df}(\mu)$ and $\mathrm{df}(\sigma)$ we made a search between 1 to 20 in steps of 1 and for $\lambda$ between -2 to 2 in steps of 0.05 . After optimizing these three parameters, optimal $d f(v)$ and $d f(T)$ were searched ranging between 0 to 9 in steps of 1 , respectively. In last step of procedure, fine tuning was used for the model with optimum parameters with changing values of $\mathrm{df}(\sigma)$, $\operatorname{df}(\mu), d f(v), d f(T)$ and $\lambda$. Same procedure was followed for LMST and LMS methods, considering the absence of $T$ parameter in BCCG distribution of LMS method. Results from the Table 1 demonstrates the best fit models for agerelated FFM, FFMI, FM and FMI data for both boys and girls. The contribution of fat and fat free mass (FMI, FFMI) to BMI and fat\% is shown in Hattori chart (Figure 1). ${ }^{21}$

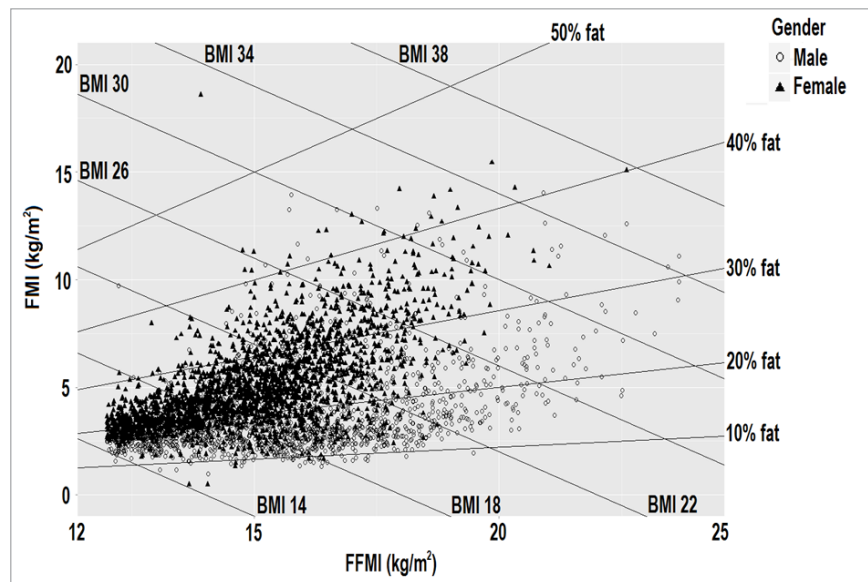

Figure 1. The contribution of fat and fat free mass (FMI, FFMI) to $\mathrm{BMI}$ and fat\% inHattori chart. Lines in this chart represents BMI and Fat $\%$. This configuration shows gender specific configuration of fat distribution in a certain BMI range. Empty dots represents males and filled triangels represents females.

\section{Results}

The LMSP method parameters used to produce FM, FFM, FMI and FFMI. Our initial observation for FMI and FM was female predominance through 6 to 17 years in which the predominance significantly increases after 9 years (Table 1). The gender differences in 6 years were
$0.16 \mathrm{~kg}$ but in 17 years gender difference became 2.05 $\mathrm{kg}$. Although FM increased gradually from 6 to 17 years in male gender, there was a peak of FM in 14 years of female which then relatively decreases (Table 1).

The differences in $3^{\text {rd }}-97^{\text {th }}$ percentiles of FM were 4.38 kg-19.65 kg respectively, for 6 and 17 years old males where this difference was $5.57-19.65 \mathrm{~kg}$ at $6-17$ years old females (Table 2). The increase in FM through 6-17 years old was $2.54 \mathrm{~kg}$ and $2.94 \mathrm{~kg}$ for 3rd percentile and 17.81 and 17.02 for $97^{\text {th }}$ percentiles respectively for males and females. The $50^{\text {th }}$ percentile increases for $50^{\text {th }}$ percentile through 6-17 years were 5.85 and 8.57 respectively for males and females (Table 2).

The differences in $3^{\text {rd }}-97^{\text {th }}$ percentiles of FMI were 3.15 $6.44 \mathrm{~kg} / \mathrm{m}^{2}$ respectively for 6 and 17 years old male where this difference was $3.38-7.59 \mathrm{~kg} / \mathrm{m}^{2}$ at $6-17$ years old females (Table 3 ). The increase in FMI through $6-17$ years old was $-0.49 \mathrm{~kg} / \mathrm{m}^{2}$ and $0.8 \mathrm{~kg} / \mathrm{m}^{2}$ for $3^{\text {rd }}$ percentile and $2.8 \mathrm{~kg} / \mathrm{m}^{2}$ and $4.29 \mathrm{~kg} / \mathrm{m}^{2}$ for $97^{\text {th }}$ percentile respectively for males and females. The $50^{\text {th }}$ percentile increases for $50^{\text {th }}$ percentile through $6-17$ years were 0.17 and $2.84 \mathrm{~kg} /$ $\mathrm{m}^{2}$ respectively, for males and females (Table 3 ). In Table 4, we found that the mean FFM was slightly higher than females, but significantly higher after 13 years.

The differences in $3^{\text {rd }}-97^{\text {th }}$ percentiles of FFM were 8.16 $\mathrm{kg}-27.06 \mathrm{~kg}$ respectively, for 6 and 17 years old males where this difference was $7.95-13.31 \mathrm{~kg}$ at $6-17$ years old females (Table 5). The increase in FFM through 6-17 years old was $30.05 \mathrm{~kg}$ and $23.63 \mathrm{~kg}$ for 3 rd percentile and 48.95 and 28.99 for $97^{\text {th }}$ percentile respectively, for males and females. The $50^{\text {th }}$ percentile increases for $50^{\text {th }}$ percentile through 6-17 years were 34.77 and 25.83 respectively for males and females (Table 5).

The difference in $3^{\text {rd }}-97^{\text {th }}$ percentiles of FFMI were 4.06 $\mathrm{kg} / \mathrm{m}^{2}-7.20 \mathrm{~kg} / \mathrm{m}^{2}$ respectively for 6 and 17 years old males, where this difference was $4.06-6.95 \mathrm{~kg} / \mathrm{m}^{2}$ at 6-17 years old females (Table 6). The increase in FFMI through $6-17$ years old was $4.06 \mathrm{~kg} / \mathrm{m}^{2}$ and $4.06 \mathrm{~kg} / \mathrm{m}^{2}$ for $3^{\text {rd }}$ percentile and $7.20 \mathrm{~kg} / \mathrm{m}^{2}$ and $6.95 \mathrm{~kg} / \mathrm{m}^{2}$ for $97^{\text {th }}$ percentiles respectively, for males and females. The $50^{\text {th }}$ percentile increases for $50^{\text {th }}$ percentile through $6-17$ years were $5.25 \mathrm{~kg} / \mathrm{m}^{2}$ and $4.67 \mathrm{~kg} / \mathrm{m}^{2}$ respectively, for males and females (Table 6).

\begin{tabular}{|c|c|c|c|c|c|c|}
\hline \multirow[b]{2}{*}{ Age (years) } & \multicolumn{3}{|c|}{ Boys } & \multicolumn{3}{|c|}{ Girls } \\
\hline & n & $\begin{array}{c}\text { Fat mass index } \\
\text { Mean (SD) }\end{array}$ & $\begin{array}{l}\text { Fat Mass } \\
\text { Mean (SD) }\end{array}$ & $\mathbf{n}$ & $\begin{array}{c}\text { Fat mass index } \\
\text { Mean (SD) }\end{array}$ & $\begin{array}{c}\text { Fat Mass } \\
\text { Mean (SD) }\end{array}$ \\
\hline 6 & 119 & $3.26(1.10)^{*}$ & $4.63(1.68)$ & 129 & $3.52(0.97)$ & $4.79(1.44)$ \\
\hline 7 & 148 & $3.53(1.30)$ & $5.42(2.12)$ & 161 & $3.82(1.36)$ & $5.84(2.15)$ \\
\hline 8 & 160 & $3.71(1.56)$ & $6.32(2.83)$ & 165 & $3.89(1.48)$ & $6.52(2.85)$ \\
\hline 9 & 141 & $4.06(2.13)$ & $7.49(4.11)$ & 144 & $4.20(1.61)$ & $7.56(3.15)$ \\
\hline 10 & 163 & $3.87(1.83)^{*}$ & $7.71(3.97)^{*}$ & 177 & $4.70(1.92)$ & $9.57(4.27)$ \\
\hline 11 & 141 & $3.83(1.92)^{*}$ & $8.08(4.40)^{*}$ & 115 & $4.78(1.90)$ & $10.48(4.57)$ \\
\hline 12 & 116 & $3.98(2.18)^{*}$ & $9.09(5.23)^{*}$ & 156 & $4.87(1.69)$ & $11.53(4.32)$ \\
\hline 13 & 133 & $3.72(1.90)^{*}$ & $9.31(4.72)^{\star}$ & 159 & $5.55(2.39)$ & $13.46(5.66)$ \\
\hline 14 & 147 & $3.56(2.04)^{*}$ & $9.92(5.91)^{*}$ & 147 & $6.05(2.17)$ & $15.58(6.03)$ \\
\hline 15 & 217 & $3.53(1.73)^{*}$ & $10.48(5.20)^{*}$ & 378 & $5.35(2.04)$ & $13.85(5.42)$ \\
\hline 16 & 212 & $3.79(2.05)^{*}$ & $11.47(6.11)^{*}$ & 413 & $5.56(2.27)$ & $14.41(5.98)$ \\
\hline 17 & 84 & $3.74(1.72)^{*}$ & $11.44(5.29)^{*}$ & 133 & $5.26(1.96)$ & $13.49(4.96)$ \\
\hline
\end{tabular}




\begin{tabular}{|c|c|c|c|c|c|c|c|c|c|c|c|}
\hline \multirow{2}{*}{$\begin{array}{l}\begin{array}{l}\text { Age } \\
\text { (years) }\end{array} \\
\end{array}$} & \multicolumn{11}{|c|}{ Percentiles } \\
\hline & $3^{\text {rd }}$ & $5^{\text {th }}$ & $10^{\text {th }}$ & $15^{\text {th }}$ & $25^{\text {th }}$ & $50^{\text {th }}$ & $75^{\text {th }}$ & $85^{\text {th }}$ & $90^{\text {th }}$ & $95^{\text {th }}$ & $97^{\text {th }}$ \\
\hline \multicolumn{12}{|l|}{ Boys } \\
\hline 6 & 2.64 & 2.75 & 2.93 & 3.07 & 3.30 & 3.82 & 4.54 & 5.07 & 5.51 & 6.33 & 7.02 \\
\hline 7 & 2.95 & 3.09 & 3.33 & 3.51 & 3.82 & 4.59 & 5.71 & 6.53 & 7.20 & 8.46 & 9.51 \\
\hline 8 & 3.16 & 3.32 & 3.60 & 3.83 & 4.22 & 5.27 & 6.86 & 8.01 & 8.97 & 10.72 & 12.17 \\
\hline 9 & 3.30 & 3.48 & 3.81 & 4.08 & 4.56 & 5.87 & 7.93 & 9.43 & 10.65 & 12.88 & 14.70 \\
\hline 10 & 3.44 & 3.65 & 4.02 & 4.32 & 4.88 & 6.42 & 8.88 & 10.64 & 12.08 & 14.66 & 16.72 \\
\hline 11 & 3.62 & 3.84 & 4.26 & 4.60 & 5.21 & 6.94 & 9.68 & 11.64 & 13.22 & 16.03 & 18.25 \\
\hline 12 & 3.83 & 4.08 & 4.53 & 4.90 & 5.57 & 7.43 & 10.38 & 12.48 & 14.16 & 17.16 & 19.52 \\
\hline 13 & 4.09 & 4.36 & 4.84 & 5.24 & 5.95 & 7.92 & 11.00 & 13.20 & 14.96 & 18.10 & 20.58 \\
\hline 14 & 4.37 & 4.66 & 5.18 & 5.59 & 6.34 & 8.38 & 11.56 & 13.83 & 15.66 & 18.93 & 21.52 \\
\hline 15 & 4.66 & 4.96 & 5.51 & 5.95 & 6.73 & 8.83 & 12.09 & 14.43 & 16.34 & 19.78 & 22.53 \\
\hline 16 & 4.93 & 5.25 & 5.82 & 6.28 & 7.10 & 9.26 & 12.61 & 15.04 & 17.04 & 20.70 & 23.67 \\
\hline 17 & 5.18 & 5.52 & 6.12 & 6.60 & 7.45 & 9.67 & 13.10 & 15.63 & 17.74 & 21.63 & 24.83 \\
\hline \multicolumn{12}{|l|}{ Girls } \\
\hline 6 & 3.05 & 3.17 & 3.39 & 3.56 & 3.84 & 4.53 & 5.51 & 6.21 & 6.77 & 7.79 & 8.62 \\
\hline 7 & 3.08 & 3.21 & 3.45 & 3.63 & 3.94 & 4.75 & 5.93 & 6.74 & 7.40 & 8.58 & 9.51 \\
\hline 8 & 3.57 & 3.73 & 4.02 & 4.25 & 4.65 & 5.72 & 7.32 & 8.42 & 9.29 & 10.81 & 12.01 \\
\hline 9 & 3.72 & 3.90 & 4.22 & 4.48 & 4.95 & 6.22 & 8.14 & 9.45 & 10.46 & 12.22 & 13.56 \\
\hline 10 & 4.25 & 4.48 & 4.88 & 5.21 & 5.80 & 7.44 & 9.91 & 11.55 & 12.81 & 14.94 & 16.53 \\
\hline 11 & 4.93 & 5.21 & 5.73 & 6.15 & 6.90 & 8.97 & 12.04 & 14.04 & 15.54 & 18.03 & 19.84 \\
\hline 12 & 5.34 & 5.69 & 6.30 & 6.80 & 7.69 & 10.06 & 13.45 & 15.62 & 17.22 & 19.84 & 21.70 \\
\hline 13 & 5.88 & 6.30 & 7.05 & 7.65 & 8.70 & 11.41 & 15.15 & 17.49 & 19.21 & 21.98 & 23.94 \\
\hline 14 & 6.54 & 7.06 & 7.98 & 8.70 & 9.95 & 13.06 & 17.21 & 19.78 & 21.66 & 24.67 & 26.79 \\
\hline 15 & 6.53 & 7.11 & 8.13 & 8.91 & 10.25 & 13.46 & 17.62 & 20.18 & 22.05 & 25.03 & 27.12 \\
\hline 16 & 6.26 & 6.88 & 7.95 & 8.77 & 10.13 & 13.31 & 17.31 & 19.77 & 21.57 & 24.43 & 26.42 \\
\hline 17 & 5.99 & 6.64 & 7.76 & 8.60 & 9.98 & 13.10 & 16.93 & 19.29 & 21.00 & 23.74 & 25.64 \\
\hline
\end{tabular}

\section{Table 3}

Fat mass index percentiles of Turkish children and adolescents

\begin{tabular}{|c|c|c|c|c|c|c|c|c|c|c|c|}
\hline \multirow{2}{*}{$\begin{array}{l}\text { Age } \\
\text { (years) }\end{array}$} & \multicolumn{11}{|c|}{ Percentiles } \\
\hline & $3^{\text {rd }}$ & $5^{\text {th }}$ & $10^{\text {th }}$ & $15^{\text {th }}$ & $25^{\text {th }}$ & $50^{\text {th }}$ & $75^{\text {th }}$ & $85^{\text {th }}$ & $90^{\text {th }}$ & $95^{\text {th }}$ & $97^{\text {th }}$ \\
\hline \multicolumn{12}{|l|}{ Boys } \\
\hline 6 & 2.12 & 2.20 & 2.33 & 2.43 & 2.60 & 2.98 & 3.52 & 3.90 & 4.21 & 4.79 & 5.27 \\
\hline 7 & 2.09 & 2.18 & 2.34 & 2.46 & 2.65 & 3.12 & 3.80 & 4.30 & 4.72 & 5.52 & 6.21 \\
\hline 8 & 2.04 & 2.14 & 2.31 & 2.45 & 2.67 & 3.21 & 4.00 & 4.61 & 5.12 & 6.11 & 6.98 \\
\hline 9 & 1.97 & 2.08 & 2.26 & 2.41 & 2.65 & 3.25 & 4.13 & 4.80 & 5.37 & 6.49 & 7.45 \\
\hline 10 & 1.90 & 2.01 & 2.21 & 2.36 & 2.62 & 3.25 & 4.19 & 4.91 & 5.51 & 6.68 & 7.69 \\
\hline 11 & 1.84 & 1.95 & 2.16 & 2.31 & 2.58 & 3.24 & 4.22 & 4.96 & 5.59 & 6.79 & 7.81 \\
\hline 12 & 1.78 & 1.90 & 2.11 & 2.27 & 2.55 & 3.22 & 4.23 & 4.99 & 5.63 & 6.85 & 7.88 \\
\hline 13 & 1.74 & 1.86 & 2.07 & 2.24 & 2.52 & 3.20 & 4.23 & 5.00 & 5.65 & 6.88 & 7.92 \\
\hline 14 & 1.70 & 1.83 & 2.04 & 2.20 & 2.49 & 3.19 & 4.23 & 5.01 & 5.67 & 6.91 & 7.95 \\
\hline 15 & 1.67 & 1.80 & 2.01 & 2.18 & 2.47 & 3.17 & 4.23 & 5.02 & 5.69 & 6.94 & 7.99 \\
\hline 16 & 1.65 & 1.77 & 1.99 & 2.16 & 2.45 & 3.16 & 4.23 & 5.03 & 5.70 & 6.97 & 8.03 \\
\hline 17 & 1.63 & 1.75 & 1.97 & 2.14 & 2.43 & 3.15 & 4.23 & 5.04 & 5.72 & 7.01 & 8.07 \\
\hline \multicolumn{12}{|l|}{ Girls } \\
\hline 6 & 2.35 & 2.42 & 2.55 & 2.66 & 2.83 & 3.28 & 3.92 & 4.34 & 4.68 & 5.27 & 5.73 \\
\hline 7 & 2.33 & 2.41 & 2.56 & 2.68 & 2.88 & 3.39 & 4.13 & 4.63 & 5.02 & 5.70 & 6.23 \\
\hline 8 & 2.32 & 2.42 & 2.58 & 2.71 & 2.93 & 3.53 & 4.38 & 4.95 & 5.40 & 6.17 & 6.76 \\
\hline 9 & 2.32 & 2.42 & 2.60 & 2.74 & 2.99 & 3.66 & 4.63 & 5.28 & 5.77 & 6.61 & 7.24 \\
\hline 10 & 2.39 & 2.50 & 2.71 & 2.87 & 3.16 & 3.93 & 5.04 & 5.76 & 6.31 & 7.22 & 7.89 \\
\hline 11 & 2.48 & 2.61 & 2.85 & 3.03 & 3.36 & 4.24 & 5.49 & 6.29 & 6.88 & 7.86 & 8.56 \\
\hline 12 & 2.56 & 2.71 & 2.97 & 3.18 & 3.55 & 4.53 & 5.90 & 6.75 & 7.38 & 8.40 & 9.12 \\
\hline 13 & 2.63 & 2.80 & 3.09 & 3.33 & 3.74 & 4.81 & 6.27 & 7.18 & 7.84 & 8.90 & 9.64 \\
\hline 14 & 2.66 & 2.85 & 3.18 & 3.44 & 3.90 & 5.05 & 6.58 & 7.52 & 8.20 & 9.29 & 10.05 \\
\hline 15 & 2.62 & 2.82 & 3.19 & 3.47 & 3.95 & 5.14 & 6.70 & 7.65 & 8.34 & 9.42 & 10.18 \\
\hline 16 & 2.53 & 2.75 & 3.13 & 3.43 & 3.93 & 5.15 & 6.70 & 7.64 & 8.32 & 9.39 & 10.13 \\
\hline 17 & 2.43 & 2.66 & 3.06 & 3.37 & 3.89 & 5.12 & 6.66 & 7.58 & 8.25 & 9.30 & 10.02 \\
\hline
\end{tabular}




\begin{tabular}{lcccccc}
\hline \multirow{2}{*}{ Age (years) } & \multicolumn{2}{c}{ Boys } & \multicolumn{2}{c}{ Girls } \\
\cline { 2 - 7 } & $\mathbf{n}$ & $\begin{array}{c}\text { FFM index } \\
\text { Mean (SD) }\end{array}$ & $\begin{array}{c}\text { FFM } \\
\text { Mean (SD) }\end{array}$ & $\mathbf{n}$ & $\begin{array}{c}\text { FFM index } \\
\text { Mean (SD) }\end{array}$ & $\begin{array}{c}\text { FFM } \\
\text { Mean (SD) }\end{array}$ \\
\hline 6 & 119 & $12.84(1.09)^{*}$ & $18.20(2.35)^{*}$ & 130 & $12.16(1.01)$ & $16.49(2.24)$ \\
7 & 148 & $13.17(1.1)^{*}$ & $20.18(2.89)^{*}$ & 160 & $12.59(1.23)$ & $19.23(2.65)$ \\
8 & 160 & $13.59(1.29)^{*}$ & $22.99(3.27)^{*}$ & 166 & $12.86(1.2)$ & $21.41(3.29)$ \\
9 & 141 & $14.02(1.32)^{*}$ & $25.72(3.66)^{*}$ & 145 & $13.26(1.23)$ & $25.72(3.66)$ \\
10 & 163 & $14.09(1.42)$ & $27.78(4.40)$ & 177 & $13.95(1.55)$ & $28.20(4.99)$ \\
11 & 141 & $14.64(1.56)^{*}$ & $30.62(5.07)$ & 115 & $14.22(1.62)$ & $30.95(5.09)$ \\
12 & 118 & $15.22(1.44)^{*}$ & $34.75(5.52)$ & 156 & $14.65(1.65)$ & $34.52(5.32)$ \\
13 & 133 & $15.89(1.66)^{*}$ & $39.89(6.79)^{*}$ & 157 & $15.03(1.78)$ & $36.60(4.89)$ \\
14 & 147 & $16.64(1.86)^{*}$ & $46.46(7.78)^{*}$ & 147 & $15.49(1.68)$ & $39.74(5.49)$ \\
15 & 216 & $17.30(1.83)^{*}$ & $51.56(7.21)^{*}$ & 378 & $15.85(1.34)$ & $40.86(3.79)$ \\
16 & 213 & $17.79(1.99)^{*}$ & $54.06(7.56)^{*}$ & 412 & $16.11(1.26)$ & $41.71(3.56)$ \\
17 & 84 & $18.14(1.72)^{*}$ & $55.59(6.92)^{*}$ & 133 & $15.96(1.26)$ & $41.08(3.82)$ \\
\hline
\end{tabular}

Age indicates whole age group (e.g. 7.00-7.99 years, etc.), ${ }^{*}$ p $<0.05$, SD; Standard Deviation

Table 5

Fat-free mass percentiles of Turkish children and adolescents

\begin{tabular}{|c|c|c|c|c|c|c|c|c|c|c|c|}
\hline \multirow{2}{*}{$\begin{array}{l}\text { Age } \\
\text { (years) }\end{array}$} & \multicolumn{11}{|c|}{ Percentiles } \\
\hline & $3^{\text {rd }}$ & $5^{\text {th }}$ & $10^{\text {th }}$ & $15^{\text {th }}$ & $25^{\text {th }}$ & $50^{\text {th }}$ & $75^{\text {th }}$ & $85^{\text {th }}$ & $90^{\text {th }}$ & $95^{\text {th }}$ & $97^{\text {th }}$ \\
\hline \multicolumn{12}{|l|}{ Boys } \\
\hline 6 & 13.43 & 13.82 & 14.44 & 14.87 & 15.53 & 16.79 & 18.21 & 19.10 & 19.76 & 20.83 & 21.59 \\
\hline 7 & 15.06 & 15.49 & 16.19 & 16.68 & 17.43 & 18.94 & 20.65 & 21.69 & 22.45 & 23.68 & 24.55 \\
\hline 8 & 16.90 & 17.38 & 18.17 & 18.73 & 19.60 & 21.41 & 23.48 & 24.71 & 25.61 & 27.03 & 28.01 \\
\hline 9 & 18.72 & 19.25 & 20.13 & 20.76 & 21.77 & 23.91 & 26.40 & 27.85 & 28.89 & 30.51 & 31.63 \\
\hline 11 & 22.19 & 22.82 & 23.89 & 24.68 & 25.98 & 28.88 & 32.29 & 34.23 & 35.60 & 37.69 & 39.10 \\
\hline 12 & 24.45 & 25.17 & 26.37 & 27.27 & 28.75 & 32.10 & 36.04 & 38.27 & 39.83 & 42.21 & 43.81 \\
\hline 13 & 27.60 & 28.44 & 29.84 & 30.89 & 32.60 & 36.45 & 40.96 & 43.51 & 45.28 & 47.99 & 49.81 \\
\hline 14 & 31.94 & 32.94 & 34.59 & 35.81 & 37.78 & 42.11 & 47.13 & 49.98 & 51.97 & 55.02 & 57.08 \\
\hline 15 & 36.86 & 38.00 & 39.88 & 41.24 & 43.40 & 48.01 & 53.29 & 56.33 & 58.47 & 61.78 & 64.03 \\
\hline 16 & 40.72 & 41.93 & 43.91 & 45.33 & 47.54 & 52.13 & 57.33 & 60.39 & 62.56 & 65.97 & 68.31 \\
\hline \multicolumn{12}{|l|}{ Girls } \\
\hline 6 & 12.23 & 12.57 & 13.14 & 13.54 & 14.17 & 15.46 & 16.94 & 17.82 & 18.46 & 19.48 & 20.18 \\
\hline 7 & 13.84 & 14.23 & 14.87 & 15.33 & 16.05 & 17.52 & 19.21 & 20.22 & 20.95 & 22.10 & 22.90 \\
\hline 8 & 15.62 & 16.07 & 16.81 & 17.33 & 18.15 & 19.84 & 21.78 & 22.93 & 23.76 & 25.08 & 26.00 \\
\hline 9 & 17.40 & 17.93 & 18.81 & 19.43 & 20.41 & 22.43 & 24.74 & 26.13 & 27.13 & 28.71 & 29.81 \\
\hline 10 & 19.15 & 19.82 & 20.92 & 21.70 & 22.93 & 25.46 & 28.38 & 30.12 & 31.38 & 33.38 & 34.77 \\
\hline 11 & 21.44 & 22.23 & 23.53 & 24.46 & 25.91 & 28.88 & 32.27 & 34.29 & 35.73 & 38.01 & 39.59 \\
\hline 12 & 24.59 & 25.44 & 26.80 & 27.76 & 29.26 & 32.28 & 35.65 & 37.62 & 39.02 & 41.21 & 42.70 \\
\hline 13 & 27.70 & 28.55 & 29.92 & 30.88 & 32.37 & 35.34 & 38.62 & 40.50 & 41.84 & 43.91 & 45.31 \\
\hline 14 & 30.05 & 30.93 & 32.34 & 33.33 & 34.86 & 37.94 & 41.32 & 43.28 & 44.66 & 46.81 & 48.27 \\
\hline 15 & 32.88 & 33.67 & 34.94 & 35.83 & 37.20 & 39.95 & 42.97 & 44.71 & 45.94 & 47.85 & 49.14 \\
\hline 16 & 35.33 & 35.98 & 37.03 & 37.76 & 38.89 & 41.15 & 43.64 & 45.08 & 46.10 & 47.69 & 48.77 \\
\hline
\end{tabular}

We checked the age-specific contribution of FMI, FFMI to BMI and fat\% with Hattori chart (Figure 1). In interpretation of Hattori chart, we found that children with similar BMI may lie in different fat \%.

Another finding was discrimination between males and females for fat \% which was prominent in BMI higher than $26 \mathrm{~kg} / \mathrm{m}^{2}$. The $50^{\text {th }}$ percentiles of FM, FMI, FFM and FFMI were compared in Figure 2. The prominent findings in Figure 3 were the increase in FM from 9 years on and increase in FFM from 13 years on respectively, for females and males.

\section{Discussion}

The well-known determinant of body composition; the $\mathrm{BMI}$ is composed of both the body fat (FM) and fat-free mass (FFM). In this study, we produced both FM and FFM 


\begin{tabular}{|c|c|c|c|c|c|c|c|c|c|c|c|}
\hline \multirow{2}{*}{$\begin{array}{l}\text { Age } \\
\text { (years) }\end{array}$} & \multicolumn{11}{|c|}{ Percentiles } \\
\hline & $3^{\text {rd }}$ & $5^{\text {th }}$ & $10^{\text {th }}$ & $15^{\text {th }}$ & $25^{\text {th }}$ & $50^{\text {th }}$ & $75^{\text {th }}$ & $85^{\text {th }}$ & $90^{\text {th }}$ & $95^{\text {th }}$ & $97^{\text {th }}$ \\
\hline \multicolumn{12}{|l|}{ Boys } \\
\hline 6 & 11.02 & 11.18 & 11.44 & 11.63 & 11.92 & 12.55 & 13.29 & 13.75 & 14.10 & 14.67 & 15.08 \\
\hline 7 & 11.27 & 11.44 & 11.72 & 11.91 & 12.23 & 12.89 & 13.68 & 14.17 & 14.53 & 15.13 & 15.56 \\
\hline 8 & 11.52 & 11.70 & 12.00 & 12.21 & 12.54 & 13.24 & 14.08 & 14.59 & 14.98 & 15.60 & 16.05 \\
\hline 9 & 11.75 & 11.94 & 12.26 & 12.48 & 12.84 & 13.58 & 14.46 & 15.00 & 15.41 & 16.06 & 16.53 \\
\hline 10 & 11.97 & 12.18 & 12.51 & 12.75 & 13.13 & 13.92 & 14.85 & 15.42 & 15.84 & 16.52 & 17.00 \\
\hline 11 & 12.24 & 12.46 & 12.81 & 13.07 & 13.47 & 14.31 & 15.28 & 15.88 & 16.32 & 17.02 & 17.52 \\
\hline 12 & 12.60 & 12.83 & 13.21 & 13.49 & 13.91 & 14.80 & 15.83 & 16.46 & 16.92 & 17.65 & 18.17 \\
\hline 13 & 13.07 & 13.32 & 13.72 & 14.02 & 14.47 & 15.41 & 16.50 & 17.16 & 17.65 & 18.42 & 18.96 \\
\hline 14 & 13.60 & 13.87 & 14.30 & 14.60 & 15.08 & 16.08 & 17.24 & 17.93 & 18.44 & 19.26 & 19.84 \\
\hline 15 & 14.13 & 14.40 & 14.85 & 15.17 & 15.68 & 16.73 & 17.94 & 18.68 & 19.22 & 20.09 & 20.70 \\
\hline 16 & 14.60 & 14.89 & 15.35 & 15.68 & 16.21 & 17.30 & 18.57 & 19.35 & 19.92 & 20.84 & 21.50 \\
\hline 17 & 15.05 & 15.35 & 15.83 & 16.17 & 16.71 & 17.84 & 19.17 & 19.98 & 20.59 & 21.56 & 22.25 \\
\hline \multicolumn{12}{|l|}{ Girls } \\
\hline 6 & 11.14 & 11.30 & 11.56 & 11.75 & 12.05 & 12.68 & 13.42 & 13.88 & 14.23 & 14.79 & 15.20 \\
\hline 7 & 11.34 & 11.51 & 11.79 & 11.99 & 12.30 & 12.96 & 13.74 & 14.23 & 14.59 & 15.18 & 15.60 \\
\hline 8 & 11.56 & 11.74 & 12.03 & 12.24 & 12.58 & 13.28 & 14.11 & 14.62 & 15.00 & 15.62 & 16.07 \\
\hline 9 & 11.74 & 11.93 & 12.24 & 12.47 & 12.82 & 13.57 & 14.45 & 14.99 & 15.39 & 16.05 & 16.51 \\
\hline 10 & 11.91 & 12.11 & 12.45 & 12.69 & 13.06 & 13.85 & 14.78 & 15.35 & 15.77 & 16.46 & 16.95 \\
\hline 11 & 12.10 & 12.32 & 12.68 & 12.93 & 13.33 & 14.17 & 15.16 & 15.76 & 16.20 & 16.92 & 17.42 \\
\hline 12 & 12.46 & 12.69 & 13.07 & 13.34 & 13.77 & 14.65 & 15.69 & 16.32 & 16.78 & 17.52 & 18.04 \\
\hline 13 & 12.99 & 13.24 & 13.64 & 13.93 & 14.38 & 15.32 & 16.40 & 17.06 & 17.54 & 18.31 & 18.85 \\
\hline 14 & 13.61 & 13.87 & 14.30 & 14.61 & 15.09 & 16.08 & 17.23 & 17.92 & 18.43 & 19.24 & 19.81 \\
\hline 15 & 14.19 & 14.47 & 14.92 & 15.24 & 15.74 & 16.79 & 18.01 & 18.75 & 19.29 & 20.16 & 20.77 \\
\hline 16 & 14.64 & 14.92 & 15.39 & 15.73 & 16.25 & 17.35 & 18.64 & 19.42 & 20.00 & 20.93 & 21.59 \\
\hline 17 & 15.14 & 15.44 & 15.91 & 16.26 & 16.80 & 17.93 & 19.26 & 20.07 & 20.67 & 21.64 & 22.33 \\
\hline
\end{tabular}

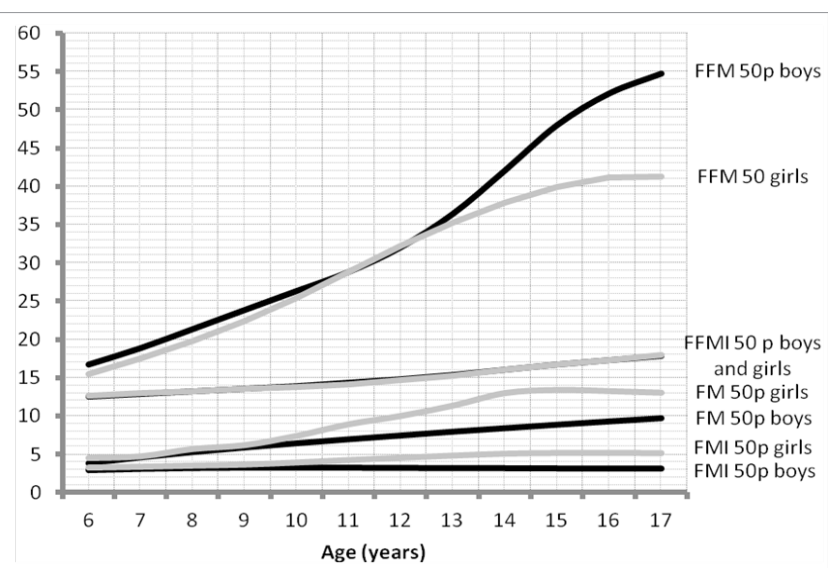

Figure 2. Gender comparison for FM, FFM, FMI and FFMI $50^{\text {th }}$ percentiles.

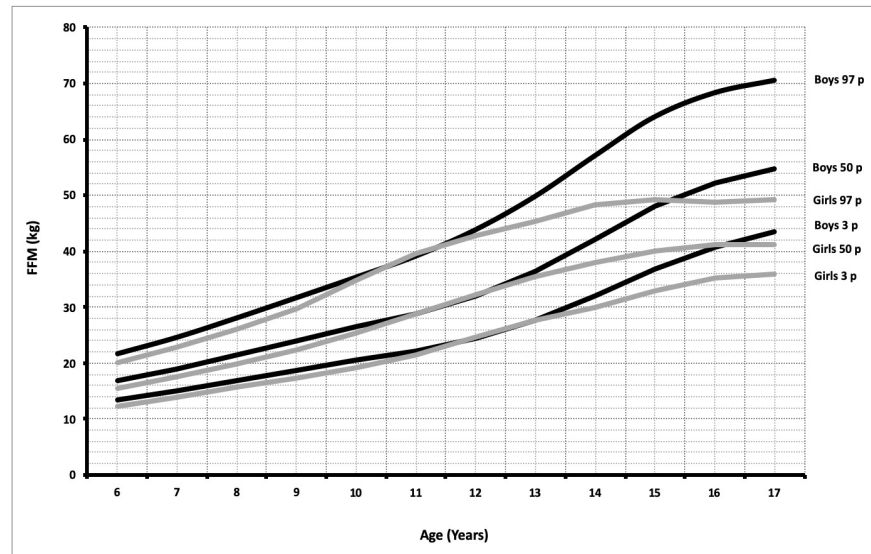

Figure 3. The comparison of $3^{\text {rd }}, 50^{\text {th }}$ anfd $97^{\text {th }}$ percentiles of FFM. references which are the two components of BMI; FMI and FFMI references of 6-17 years old Turkish children for the purpose of screen and compare these references with other references. We constructed Hattori chart in which FMI versus FFMI was classified according to both $\mathrm{BMI}$ and fat \% (Figure 1). The use of FMI, FFMI other than BMI is the fact that similar BMI may have significantly different FMI (fat mass) and FFMI (lean) component. ${ }^{22}$ In other words BMI may fail to discriminate children with overfat (fat mass) and overweight (fat mass and lean mass) both of which has discrete cardiometabolic end points.

Our findings also support the above statement by indicating that children with similar BMI may have significantly different fat content (Figure 1). Even in normal BMI range gender related splitting in favour of females for fat mass index can be observed. In the overweight children the discrimination between each gender becomes much more apparent. In overweight and obese children distraction between FMI and FFMI becomes much more clear where females are concentrated in $30-40 \%$ and males $20-30 \%$ range after the $\mathrm{BMI}$ value exceeds 18 (Figure 1). Then, we may conlude that not solely BMI but its components FMI and FFMI should be considered together in clinical assesment. Although BIA is not the gold standard it is stil may be considered as both the most practical and reliable method of screening and detection of overweight and obesity.

In comparison of our results with previous studies we detected that almost all previous studies were performed in relatively narrow age groups. Other than age group, it 
is not easy to find studies performed in similar periods which may bias comparison. FM of Turkish children was higher than Japan (2003) ${ }^{23}$ and Bengal (2013) ${ }^{24}$ children which may be related with several factors such as geographic location, nutrition, ethnic origin, and e.t.c. Considering the findings of Eissa MA et $\mathrm{al}^{25}$ in white USA children $(n=678)$ FFM ve FFMI are similar with our findings. Inconsiderable differences between our data and Eissa MA data were detected in 8, 11 and $14^{\text {th }}$ years of age in favor of USA male children and female Turkish children. This comprehensive similarity between USA and Turkish children may be an indicator of body composition change towards USA children. Comparison of our data with Bangladesh study supported our above statement indicating body composition of Turkish children is similar with Bangladesh data $(n=200)$ in relatively young children (6 years) but extending towards USA children as they get older. This finding may show that increased fat mass may be caused by nutritional shift to western type. ${ }^{26}$ Prins $\mathrm{M}$ et $\mathrm{al}^{27}$ determined FFM values in 133 Gambian children aged between 5-16 years. We detected higher FFM values for Turkish children in all ages. This finding pointed out both ethnic and socioeconomic differences. Similarly, in Nightingale CM et al. study conducted in London with Southeast Asian, African and English children aged between 8-10 years, found different FFM values and emphasized ethnicity as a determinant factor. ${ }^{28}$ McCarthy $\mathrm{HD}$ et $\mathrm{al}^{29}$ measured FFM and skeletal muscle mass with BIA in 1985 English children aged between 5-18 years. FFM values were similar to Turkish children both in males and females, whereas higher values were found in 17 years. These reflect the nutrition and physical activity difference in late adolescent period.

In another study performed with Tanita BC-418, no difference was detected between BIA-DEXA values in normal-weight children. ${ }^{13}$ In another study, the correlation of BIA and DEXA methods is also shown by Wang $L$ at al. ${ }^{8}$

In addition to determining BMI solely, information about the two components of BMI; FM and FFM is essential for pediatricians to decide about future cardiometabolic risk by body fat and its distribution..$^{29,30-32}$ Anormal body fat and its distribution lead hypertension, cardiovascular disease and type 2 diabetes mellitus and named incubation period during childhood and adolescents. ${ }^{32}$ Besides a couple of childhood diseases (such as adiposity rebound, lipodystrophy, malignancy, cystic fibrosis, obesity, eating disorders) may also lead distinctive alterations in FM, FFM and bone mineral density. ${ }^{10,30,33-36}$ Even short term existance of these disorders may cause long term effects in body composition in terms of fat and lean mass. Then determination of body composition may have significant use in progress of disorders such as obesity, eating disorders, undernutrition, FFM changes in hospital stay, growth monitoring of liver transplantation, HIV patients screening the effectiveness of therapy (insulin and etc.), change in body fat content (parenteral and gastrostomy nutrition), prediction of disorder related risks (obesity), regulation of nutrition (energy and liquid need), drug dose adjustment (dialysis, growth hormone and e.t.c). . $^{31,33,34,37}$
Another consideration is that FMI and FFMI may be significant indicator of pubertal variants, normal puberty, precocious or delayed puberty. ${ }^{38,39}$ In girls with premature adrenarche, changes in body composition as increase total body fat, decrease FFM, muscle mass and total body water were observed by Cebeci AN et al. ${ }^{39}$ Throughout male puberty, there is a progressive in total body bone mineral content, and lean body and a progressive decrease in body fat. In girls, increased in body fat content, lean body mass and body fat distribution occurs in the typical female contours. ${ }^{38}$ In our study, the prominent increase in $50^{\text {th }}$ percentile $F M$ in females after 9.5 years and increase in $50^{\text {th }}$ percentile FFM in males supports our consideration that fat to lean mass ratio significantly and inversly changes during adolescence (Figure 2).

The incorconcordence between fat mass and lean mass is classified by Shultz ${ }^{40}$ as leanness (Low FFMI vs low BMI), obesity (Low FFMI vs high FMI), muscle hypertrophy (High FFMI vs low FMI), and combined excess (High FFMI vs high FMI).

In conclusion both in screening and clinical practice use of FMI and FFMI together with BMI would significantly contribute to detect and follow-up of adiposity rebound, puberty precous, delayed puberty, overweight and obesity. This study would contribute to literature as one of the most comprehensive one. Additionally future studies on the same subject in Turkish population can use our data as acomparison base.

Ethics Committee Approval: The Ethical Committee of Erciyes University, Faculty of Medicine, approved this study (Approval date: 03.04.2019/304, number: 0401/168).

Informed Consent: Written informed consent was obtained from patients who participated in this study.

Peer-review: Externally peer-reviewed.

Author Contributions: All of the authors declare that they have all participated in the design, execution, and analysis of the paper, and that they have approved the final version

Conflict of Interest: The authors have no conflict of interest to declare.

Financial Disclosure: The authors declared that this study has received no financial support.

\section{References}

1. Lobstein T, Baur L, Uauy R. IASO International Obesity Task Force. Obesity in children and young people: a crisis in public health. Obes Rev. 2004; 5: 4-104. [CrossRef]

2. Han JC, Lawlor DA, Kimm SY. Childhood obesity. Lancet. 2010; 375: 1737-1748. [CrossRef]

3. Ernst MA, Gerver WJ, Simons MY, et al. Obesity in childhood: a secular trend or an epidemic disease? J Child Health Care. 2012; 17: 30-40. [CrossRef]

4. Johnson W, Chumlea WC, Czerwinski SA, et al. Secular trends in the fat and fat-free components of body mass index in children aged 8-18 years born 1958-1995. Ann Hum Biol. 2013; 40: 107110. [CrossRef]

5. Sabin MA, Kao KT, Juonala M, et al. Viewpoint article: Childhood obesity - looking back over 50 years to begin to look forward. $J$ Paediatr Child Health. 2015; 51: 82-86. [CrossRef] 
6. Dietz WH. Health consequences of obesity in youth: childhood predictors of adult disease. Pediatrics. 1998; 101: 518-525. [CrossRef]

7. Melanson KJ, Mcinnis KJ, Rippe JM, et al. Obesity and cardiovascular disease risk: research update. Cardiol Rev. 2001; 9: 202-207. [CrossRef]

8. Wang L, Hui, SS, Wong SH. Validity of bioelectrical impedance measurement in predicting fat-free mass of Chinese children and adolescents. Med Sci Monit. 2014; 20: 2298-2310. [CrossRef]

9. Maynard LM, Wisemandle W, Roche AF, et al. Childhood body composition in relation to body mass index. Pediatrics. 2001; 107 344-350. [CrossRef]

10. Prentice AM, Jebb SA. Beyond body mass index. Obes Reviews. 2001; 2: 141-147. [CrossRef]

11. Thibault R, Pichard $C$. The evaluation of body composition: a useful tool for clinical practice. Ann Nutr Metab. 2012; 60: 6-16. [CrossRef]

12. Aguirre CA, Salazar GD, Lopez de Romaña DV, et al. Evaluation of simple body composition methods: assessment of validity in prepubertal Chilean children. Eur J Clin Nutr. 2015; 69: 269-273. [CrossRef]

13. Meredith-Jones KA, Williams SM, Taylor RW. Bioelectrical impedance as a measure of change in body composition in young children. Pediatr Obes. 2015; 120: 252-259. [CrossRef]

14. Micklesfield LK, Goedecke JH, Punyanitya M, et al. Dual-energy X-ray performs as well as clinical computed tomography for the measurement of visceral fat. Obesity (Silver Spring). 2012; 20: 1109-1194. [CrossRef]

15. Achamrah N, Colange G, Delay J, et al. Comparison of body composition assessment by DXA and BIA according to the body mass index: A retrospective study on 3655 measures. PLoS One. 2018; 13(7): e0200465. [CrossRef]

16. Talma $\mathrm{H}$, Chinapaw MJ, Bakker B, et al. Bioelectrical impedance analysis to estimate body composition in children and adolescents: a systematic review and evidence appraisal of validity, responsiveness, reliability and measurement error. Obes Rev. 2013; 14: 895-905. [CrossRef]

17. Kurtoglu S, Mazicioglu MM, Ozturk A, et al. Body fat reference curves for healthy Turkish children and adolescents. Eur J Pediatr. 2010; 169: 1329-1335. [CrossRef]

18. Van Buuren S, Hayes DJ, Stasinopoulos DM, et al. Estimating regional centile curves from mixed data sources and countries. Stat Med. 2009; 28: 2891-2911. [CrossRef]

19. Rigby RA, Stasinopoulos DM. Generalized additive models for location, scale and shape. J Roy Stat Soc C Appl Stat. 2005; 54: 507-554. [CrossRef]

20. Rigby RA, Stasinopoulos DM. Smooth centile curves for skew and kurtotic data modelled using the Box-Cox power exponential distribution. Stat Med. 2004; 23: 3053-3076. [CrossRef]

21. Hattori K, Tatsumi N, Tanaka S. Assessment of body composition by using a new chart method. Am J Hum Biol. 1997; 9: 573-578. [CrossRef]

22. Wells JCK. The Evolutionary Biology of Human Body Fitness. Cambridge: Cambridge University Press; 2010:16-48. [CrossRef]

23. Nakao T, Komiya S. Reference norms for a fat-free mass index and fat massindex in the Japanese child population. J Physiol Anthropol Appl Hum Sci. 2003; 22: 293-298. [CrossRef]
24. Sen J, Mondal N. Fat mass and fat-free mass as indicators of body composition among Bengalee Muslim children. Ann Hum Biol. 2013; 40: 286-293. [CrossRef]

25. Eissa MA, Dai S, Mihalopoulos NL, et al. Trajectories of fat mass index, fat free-mass index, and waist circumference in children: Project HeartBeat! Am J Prev Med. 2009; 37: 34-39. [CrossRef]

26. Khan Al, Hawkesworth S, Hawlader MD, et al. Body composition of Bangladeshi children: comparison and development of leg-toleg bioelectrical impedance equation. J Health Popul Nutr. 2012; 30: 281-290. [CrossRef]

27. Prins $M$, Hawkesworth $S$, Wrigh A, et al. Use of bioelectrical impedance analysis to assess body composition in rural Gambian children. Eur J Clin Nutr. 2008; 62: 1065-1074. [CrossRef]

28. Nightingale CM, Rudnicka AR, Owen CG, et al. Are ethnic and gender specific equations needed to derive fat free mass from bioelectrical impedance in children of South asian, black-african Caribbean and white European origin? Results of the assessment of body composition in children study. PloS One. 2013; 8: e76426. [CrossRef]

29. McCarthy HD, Samani-Radia D, Jebb SA, et al. Skeletal muscle mass reference curves for children and adolescents. Pediatr Obes. 2014; 9: 249-259. [CrossRef]

30. Wells JC, Fewtrell MS. Is body composition important for paediatricians? Arch Dis Child. 2008; 93: 168-172. [CrossRef]

31. Wells JC. Toward body composition reference data for infants, children, and adolescents. Adv Nutr. 2014; 5: 320-329. [CrossRef]

32. Rose G. Incubation period of coronary heart disease. Br Med $\mathrm{J}$ (Clin Res Ed). 1982; 284: 1600-1601. [CrossRef]

33. Gaynor EP, Sullivan PB. Nutritional status and nutritional management in children with cancer. Arch Dis Child. 2015; 100: 1169-1172. [CrossRef]

34. Alicandro G, Battezzati A, Bianchi ML, et al. Estimating body composition from skinfold thicknesses and bioelectrical impedance analysis in cystic fibrosis patients. J Cyst Fibros. 2015; 14: 784-791. [CrossRef]

35. Plachta-Danielzik S, Bosy-Westphal A, Kehden B, et al. Adiposity rebound is misclassified by BMI rebound. Eur J Clin Nutr. 2013; 67: 984-989. [CrossRef]

36. Demerath EW, Schubert CM, Maynard LM, et al. Do changes in body mass index percentile reflect changes in body composition in children? Data from the Fels Longitudinal Study. Pediatrics. 2006; 117: 487-495. [CrossRef]

37. Thibault R, Genton L, Pichard C. Body composition: why, when and for who? Clin Nutr. 2012; 31: 435-447. [CrossRef]

38. Rico H, Revilla M, Villa LF, et al. Body composition in children and Tanner's stages: a study with dual-energy x-ray absorptiometry. Metabolism. 1993; 42: 967-970. [CrossRef]

39. Cebeci AN, Taş A. Higher body fat and lower fat-free mass in girls with premature adrenarche. J Clin Res Pediatr Endocrinol. 2015; 7: 45-48. [CrossRef]

40. Schutz Y, Kyle UU, Pichard C. Fat-free mass index and fat mass index percentiles in Caucasians aged 18-98 y. Int J Obes Relat Metab Disord. 2012; 26: 953-960. [CrossRef] 